\title{
EXTENSION AND INVERSION OF EXTENDED ORTHOMORPHISMS ON RIESZ SPACES
}

\author{
MICHEL DUHOUX and MATHIEU MEYER
}

(Received 12 July 1982, Revised 21 February 1983)

Communicated by R. O. Vyborny

\begin{abstract}
Let $E$ be an Archimedean Riesz space and let $\operatorname{Orth}^{\infty}(E)$ be the $f$-algebra consisting of all extended orthomorphisms on $E$, that is, of all order bounded linear operators $T: D \rightarrow E$, with $D$ an order dense ideal in $E$, such that $T(B \cap D) \subseteq B$ for every band $B$ in $E$. We give conditions on $E$ and on a Riesz subspace $F$ of $E$ insuring that every $T \in \operatorname{Orth}^{\infty}(F)$ can be extended to some $\tilde{T} \in \operatorname{Orth}^{\infty}(E)$, and we also consider the problem of inversing an extended orthomorphism on its support. The same problems are also studied in the case of $\sigma$-orthomorphisms, that is, extended orthomorphisms with a super order dense domain. Furthermore, some applications are given.
\end{abstract}

1980 Mathematics subject classification (Amer. Math. Soc.): 06 F 20, 46 A 40, 47 H 07.

Keywords and phrases: Archimedean Riesz spaces, order dense ideals, orthomorphisms, $f$-algebras.

\section{Introduction}

An extended orthomorphism on an Archimedean Riesz space $E$ is an order bounded linear mapping $T: D \rightarrow E$, where $D$ is an order dense ideal in $E$, such that $T(B \cap D) \subseteq B$ for every band $B$ in $E$. The set $\operatorname{Orth}^{\infty}(E)$ of all extended orthomorphisms on $E$ is a laterally complete Archimedean Riesz space and, even, an $f$-algebra with unit. The space $\operatorname{Orth}(E)$ of all orthomorphisms on $E$, that is, of everywhere defined extended orthomorphisms, is an $f$-subalgebra of $\operatorname{Orth}^{\infty}(E)$. Extended orthomorphisms have been introduced in a special setting by Nakano [11], used by Luxemburg and Schep [7] and were recently studied by the authors [3]. They form a natural generalization of the orthomorphisms and the richness of their structure allows to characterize many properties of $E$.

(C) 1984 Australian Mathematical Society 0263-6115/84\$A2.00+0.00 
In Section 1 we introduce the main notations and some useful tools. Section 2 is mainly devoted to the following problem: given a Riesz subspace $F$ of $E$ and $T \in \operatorname{Orth}^{\infty}(F)$, does there exist some $\tilde{T} \in \operatorname{Orth}^{\infty}(E)$ extending $T$ in some sense? The same kind of problem was considered by Wickstead [16] in the case of orthomorphisms; although $\operatorname{Orth}^{\infty}(E)$ has generally much more elements than $\operatorname{Orth}(E)$, the extension of extended orthomorphisms does not require so strong hypothesis as the extension of orthomorphisms does and, in fact, the two problems and their solutions are very different. The concept of a quasi-unital Riesz subspace in an $f$-algebra, introduced here, seems to be specially useful in that setting. The main extension theorem (2.5) is general enough to contain the case when $F$ is a quasi-unital Riesz subspace in an $f$-algebra $E(2.4)$ as well as the case when $F$ is any Riesz subspace in a uniformly complete Riesz space $E(2.6)$. Finally we use our results to study the equality $\operatorname{Orth}^{\infty}(\operatorname{Orth}(E))=\operatorname{Orth}^{\infty}(E)(2.7$, 2.10 ), which is not always true unlike the following easier ones: $\operatorname{Orth}(\operatorname{Orth}(E))=$ $\operatorname{Orth}(E)$ and $\operatorname{Orth}^{\infty}\left(\operatorname{Orth}^{\infty}(E)\right)=\operatorname{Orth}^{\infty}(E)$.

Section 3 is concerned with the invertibility of extended orthomorphisms. It is shown (3.3) that under some conditions on principal ideals of $E$, every extended orthomorphism is invertible on its support. In particular, if $E$ is uniformly complete, then $T$ is invertible in $\operatorname{Orth}^{\infty}(E)$ if and only if $|T|$ is a weak order unit (3.4).

In Section 4 we investigate the extension and inversion properties of $\sigma$-orthomorphisms, that is, of extended orthomorphisms with a super order dense domain. It is shown in $[3,4]$ that the study of the $f$-subalgebra $\operatorname{Orth}^{\sigma}(E)$ of $\operatorname{Orth}^{\infty}(E)$, consisting of all $\sigma$-orthomorphisms on $E$, allows to characterize the universal and the lateral $\sigma$-completions of many almost Dedekind $\sigma$-complete Riesz spaces.

The authors wish to express their gratitude to the referees for all their valuable suggestions.

\section{Preliminaries}

For terminology, notations and the general theory of Riesz spaces (resp. $f$-algebras, extended orthomorphisms), we refer to $[1,8]$ (resp. $[5,6,10,12],[2,3$, 7]). Nevertheless we recall here some definitions and results (with some proofs for the sake of completeness).

The symbols $i(A)$ and $b(A)$ (or $i_{E}(A)$ and $b_{E}(A)$ ) will denote respectively the ideal and the band generated in a Riesz space $E$ by a subset $A$ of $E$; if $A=\{x\}$ we shall write $i(x)$ and $b(x)$. 
An $f$-algebra is a Riesz space $E$ which is also an algebra such that $x y \geqslant 0$ for all $x, y \in E_{+}$, and such that $x z \wedge y=z x \wedge y=0$ for all $x, y, z \in E_{+}$satisfying $x \wedge y=0$. It is known that every Archimedean $f$-algebra is automatically commutative. An $f$-algebra will be called nowhere trivial if $x^{2}=0$ implies $x=0$ or, equivalently in the Archimedean case, if $x y=0$ is equivalent to $x \perp y$ (note that $x \perp y \Rightarrow x y=0$ holds in any $f$-algebra). An $f$-subalgebra is a Riesz subspace which is also a subalgebra. An f-homomorphism between two $f$-algebras is a Riesz homomorphism which is also a ring homomorphism, and similarly for $f$-isomorphism. By an ideal, even in an $f$-algebra, we shall always mean an order ideal, that is, a solid Riesz subspace.

We now recall some facts about extended orthomorphisms on an Archimedean Riesz space $E$. An extended orthomorphism on $E$ is an order bounded linear mapping $T: D_{T} \rightarrow E$, with $D_{T}$ an order dense ideal in $E$, such that $T x \perp y$ for all $x \in D_{T}$ and all $y \in E$ satisfying $x \perp y$ or, equivalently, such that $T\left(B \cap D_{T}\right) \subseteq B$ for every band $B$ in $E$. Such a $T$ can be shown to be order continuous and there is thus a largest ideal $D_{T}^{m}$ of $E$, called the maximal domain of $T$, such that $T$ can be (uniquely) extended to an extended orthomorphism $T: D_{T}^{m} \rightarrow E$.

Let $\operatorname{Orth}^{\infty}(E)$ be the set of all equivalence classes of extended orthomorphisms $S, T, \ldots$ on $E, S$ and $T$ being equivalent if $S=T$ on some order dense ideal of $E$ (this implies that $S=T$ on $D_{S}^{m}=D_{T}^{m}$ ); $D_{T}$ will denote any order dense ideal of $E$ on which $T$ can be defined. It is known that $\operatorname{Orth}^{\infty}(E)$ is a laterally complete (that is, every positive disjoint system has a supremum) Archimedean Riesz space under pointwise definitions of addition, scalar multiplication and ordering on order dense ideals of $E$. For every $S, T \in \operatorname{Orth}^{\infty}(E)$ we have the formulas:

$$
\begin{gathered}
(S \vee T) x=S x \vee T x \quad\left(0 \leqslant x \in D_{S} \cap D_{T}\right), \\
|T|(|x|)=|T(|x|)|=|T x| \quad\left(x \in D_{T}\right) .
\end{gathered}
$$

Note that the identity $I$ on $E$ is a weak order unit in $\operatorname{Orth}^{\infty}(E): T=\sup \{T \wedge n I$; $n=1,2, \ldots\}$ holds for every $T \in \operatorname{Orth}_{+}^{\infty}(E)$, since $T x \in b(x)$ implies that $T x=\sup _{n} T x \wedge n x\left(0 \leqslant x \in D_{T}\right)$.

Lemma 1.1 [3; 1.3]. If $T \in \operatorname{Orth}^{\infty}(E)$, then $D=\left\{x \in D_{T} ; T x \in i(x)\right\}$ is a super order dense ideal in $D_{T}$.

Proof. We can assume $T \geqslant 0$. Since $D=\cup_{n=1}^{+\infty} \operatorname{Ker}(T-n I)^{+}, D$ is clearly an ideal. For every $0 \leqslant x \in D_{T}$ we have $x=\sup _{n}\left(x-n^{-1} T x\right)^{+}$, and the proof will be complete if we show that $\left(x-n^{-1} T x\right)^{+} \in D(n=1,2, \ldots)$. Fix $0 \leqslant x \in D_{T}$, $0<\lambda \in \mathbf{R}$, and define $y=(x-\lambda T x)^{+}$, and $S=I-\lambda T$. In order to see that $T y \leqslant \lambda^{-1} y$ (and so that $y \in D$ ), observe first that $S^{-} \circ S^{+}: D_{T} \rightarrow E$ is defined. 
For every $0 \leqslant u \in D_{T}$ we have $S^{-}\left(S^{+} u \wedge n u\right) \wedge S^{+} u=0$, hence $S^{-}\left(S^{+} u \wedge\right.$ $n u) \wedge S^{-} S^{+} u=0$. But $S^{+} u \wedge n u \uparrow_{n} S^{+} u$ and, using the order continuity of $S^{-}$, we get $S^{-} S^{+} \wedge S^{-} S^{+}=0$, that is, $S^{-} S^{+}=0$. The required inequality $T y \leqslant \lambda^{-1} y$ follows now from $0=S^{-} S^{+} x=S^{-} y=(y-\lambda T y)^{-}$.

It follows from this lemma that, given $S, T \in \operatorname{Orth}^{\infty}(E)$, the product $S T$ can be defined (as in [7]) by $(S T) x=S(T x)$ for all $x$ in the order dense ideal $\left\{x \in D_{T}\right.$; $\left.T x \in D_{S}\right\}$ of $E$, and so $\operatorname{Orth}^{\infty}(E)$ becomes an Archimedean $f$-algebra with the identity $I$ on $E$ as (multiplicative) unit. In particular, the algebra $\operatorname{Orth}^{\infty}(E)$ is commutative.

The collection $\operatorname{Orth}(E)$ of all orthomorphisms on $E$, that is, of all $T \in \operatorname{Orth}^{\infty}(E)$ such that $D_{T}^{m}=E$, is obviously an $f$-subalgebra of $\operatorname{Orth}^{\infty}(E)$. The center $Z(E)$ of the Archimedean Riesz space $E$ is the $f$-subalgebra of $\operatorname{Orth}(E)$ consisting of all linear mappings $T: E \rightarrow E$ for which there exists $\lambda \in \mathbf{R}_{+}$satisfying $|T x| \leqslant \lambda x$ for all $x \in E_{+} ; Z(E)$ is obviously the (order) ideal generated by $I$ in $\operatorname{Orth}(E)$.

Lemma $1.2[7 ; 1.5]$. If $F$ is a Riesz subspace of the Dedekind complete Riesz space $E$, then every $T \in Z(F)$ can be extended to $T_{0} \in Z(E)$.

Proof. Given $T \in Z_{+}(F)$, there exists $\lambda \in \mathbf{R}_{+}$such that $T x \leqslant \lambda x$ for all $x \in F_{+}$; define $\rho(x)=\lambda x^{+}$for all $x \in E$. Then $\rho$ is a sublinear mapping and $T x \leqslant T\left(x^{+}\right) \leqslant \rho(x)$ holds for every $x \in F$. It follows from the Hahn-Banach theorem (in which $\mathbf{R}$ can be replaced by any Dedekind complete Riesz space [15]) that $T$ has a linear extension $T_{0}: E \rightarrow E$ such that $T_{0} x \leqslant \rho(x)$ for all $x \in E$; it is obvious that $T_{0} \in Z_{+}(E)$.

Let $E$ be any Archimedean Riesz space. If $T \in \operatorname{Orth}^{\infty}(E)$, then its support $B_{T}$ is defined to be the band in $E$ generated by $T\left(D_{T}\right)$; by order continuity, $B_{T}$ does not depend on the choice of domain $D_{\text {. }}$

LemMa 1.3 [3; 1.4]. If $T \in \operatorname{Orth}^{\infty}(E)$, then $\operatorname{Ker} T=D_{T} \cap B_{T}^{d}$. In particular: (1) $\operatorname{Ker} T$ is a band in $D_{T}$; (2) $T x \neq 0$ for all $x \neq 0$ in $D_{T} \cap B_{T}$; (3) $b(A) \cap B_{T}$ $=b(T(A))$ for all subsets $A$ of $D_{T}$.

Proof. If $x \in D_{T} \cap B_{T}^{d}$ then $x \perp T x$, hence $T x \perp T x$, that is, $T x=0$; so $D_{T} \cap B_{T}^{d} \subseteq \operatorname{Ker} T$. For the converse, let $0 \leqslant x \leqslant D_{T}$. Since $\operatorname{Ker} T$ is an ideal, there exists a net $0 \leqslant x_{\alpha}+y_{\alpha} \uparrow x$, with $0 \leqslant x_{\alpha} \in \operatorname{Ker} T$ and $0 \leqslant y_{\alpha} \in(\operatorname{Ker} T)^{d}$. By order continuity, $T\left(x_{\alpha}+y_{\alpha}\right)=T y_{\alpha}$ is order convergent to $T x$ and, since $T y_{\alpha} \in(\operatorname{Ker} T)$, this shows that $T x \in(\operatorname{Ker} T)^{d}$. Hence $B_{T} \subseteq(\operatorname{Ker} T)^{d}$, and so Ker $T \subseteq D_{T} \cap B_{T}^{d}$. 
(1) and (2) are obvious and (3) can be deduced from (2) and (1.1).

Corollary 1.4. If $S, T \in \operatorname{Orth}^{\infty}(E)$ satisfy $S x \leqslant T x$ for all $x$ in $A \subseteq D_{S} \cap D_{T}$ $\cap E_{+}$, then $S \leqslant T$ on $D_{S} \cap D_{T} \cap b(A)$.

Lemmas 1.1 and 1.3 are simple generalizations of known results on orthomorphisms (see [6]). The proof of the next theorem is rather technical and will be omitted.

THEOREM $1.5[3 ; 1.8]$. Let $\left(T_{\alpha} ; \alpha \in A\right)$ be a family in $\operatorname{Orth}_{+}^{\infty}(E)$ such that $T=\sup T_{\alpha}$ exists. Then

$$
T x=\sup \left\{T_{\alpha} y ; \alpha \in A \text { and } y \in D_{T_{\alpha}} \cap[0, x]\right\}
$$

for every $0 \leqslant x \in D_{T}$. In particular, if $0 \leqslant x \in D_{T} \cap\left(\cap_{\alpha \in A} D_{T_{\alpha}}\right)$, then $T x=$ $\sup \left\{T_{\alpha} x ; \alpha \in A\right\}$.

We now recall that multiplication operators on an Archimedean $f$-algebra $E$ are orthomorphisms. More precisely, we may consider the natural $f$-homomorphism $\varphi: E \rightarrow \operatorname{Orth}(E)$, defined by $\varphi(x)(y)=x y$ for all $x, y \in E$. It is well-known (and easy to verify) that $\varphi$ is one-to-one if and only if $E$ is nowhere trivial, and that $E$ and $\operatorname{Orth}(E)$ are $f$-isomorphic via $\varphi$ if and only if $E$ has a (multiplicative) unit. In particular $\operatorname{Orth}(\operatorname{Orth}(E))=\operatorname{Orth}(E)$ holds for any Archimedean Riesz space $E$. Since every laterally complete Archimedean Riesz space $E$ satisfies $\operatorname{Orth}^{\infty}(E)=$ $\operatorname{Orth}(E)[3 ; 2.8 .2]$, the next result is clear.

Lemma 1.6. If $E$ is any Archimedean Riesz space, then $\operatorname{Orth}^{\infty}\left(\operatorname{Orth}^{\infty}(E)\right)=$ $\operatorname{Orth}\left(\operatorname{Orth}^{\infty}(E)\right)=\operatorname{Orth}^{\infty}(E)$.

The next lemma will be also useful.

Lemma 1.7. Let $E$ be an Archimedean Riesz space with a uniformly complete center (this occurs in particular if $E$ is uniformly complete). If $T \in Z(E)$ satisfies $|T| \geqslant \lambda I$ for some $0<\lambda \in \mathbf{R}$, then $T$ has an inverse in $Z(E)$.

PROOF. By Yosida representation theorem [8, 14], $Z(E)$ is Riesz isomorphic to $\mathcal{C}(K)$, with $I=1$, for some compact space $K$. It follows easily from $I=1$ and 1.4 that this Riesz isomorphism is actually an $f$-isomorphism, and the result is now obvious. 
Given an Archimedean Riesz space $E$, let $\&$ be a Riesz subspace of $\operatorname{Orth}^{\infty}(E)$ and, for every ideal $J$ of $E$, define

$$
\mathscr{Q}(J)=\left\{T \in \mathscr{Q} ; B_{T} \subseteq J\right\} .
$$

$\mathscr{Q}(J)$ is clearly an ideal in $Q$. In the special case when $Q=\operatorname{Orth}^{\infty}(E)$, $\operatorname{Orth}(E)$ or $Z(E)$, we shall write $\operatorname{Orth}^{\infty}(E, J), \operatorname{Orth}(E, J)$ or $Z(E, J)$ instead of $\mathscr{Q}(J)$.

We shall say that $Q$ is rich if $Q(b(x)) \neq\{0\}$ for all $x \neq 0$ in $E$; by [3; 2.4], $Q$ is rich if and only if $Q$ is order dense in $\operatorname{Orth}^{\infty}(E)$.

We shall say that $\mathbb{Q}$ is ultrarich if $\mathscr{Q}(i(x)) \neq\{0\}$ for all $x \neq 0$ in $E$. Riesz spaces with a rich or ultrarich center have been studied in [9].

EXAMPLES. If $\Omega$ is a locally compact topological space and $E=\mathscr{K}(\Omega)$ is the Riesz space of all real continuous functions on $\Omega$ with compact support, then $E$ has an ultrarich center ( $f$-isomorphic to $e^{b}(\Omega)$, the bounded continuous functions on $\Omega$ operating by product). If $E=L^{p}(\mu), 1 \leqslant p<+\infty$, where $\mu$ is the Lebesgue measure on $[0,1]$, then $Z(E)$ ( $f$-isomorphic to $\left.L^{\infty}(\mu)\right)$ is rich (since $E$ is Dedekind complete), but $Z(E)$ is not ultrarich because $Z(E, i(f))=\{0\}$ for all $f \in E$ (see also $[9 ; 4.11])$.

\section{Extension theorems and applications}

The new concept that we introduce now is very important for what follows.

Let $F$ be a Riesz subspace of the Archimedean $f$-algebra $E$. We shall say that $F$ is quasi-unital if the ideal generated in $F$ by

$$
M=\left\{x \in F ; x y=x \text { for some } y \in F_{+}\right\}
$$

is order dense in $F$; if this ideal is super order dense in $F$, we shall say that $F$ is super quasi-unital. Notice that $x y=x$ in $E$ implies that $x^{\prime} y=x^{\prime}$ for all $x^{\prime} \in b_{E}(x)$ (an easy consequence of 1.4); in particular, $M$ is a solid subset of $F$. It follows easily from this and $\lambda M \subseteq M(\lambda \in \mathbf{R})$ that

$$
i_{F}(M)_{+}=\left\{\sum_{i=1}^{n} x_{i} ; 0 \leqslant x_{i} \in M \text { and } n=1,2, \ldots\right\} .
$$

Considering the following statements

(1) $E$ has a unit $e$ and $e \in F$,

(2) $E$ has a unit $e$ and $x \wedge e \in F$ for all $x \in F_{+}$,

(3) $E$ is nowhere trivial and, for every $x \in F_{+}$, there exists $u \in F_{+}$such that $x y \wedge y=u y$ for all $y \in F_{+}$,

(4) $F$ is super quasi-unital, 
it is not difficult to verify that (1) $\Rightarrow(2) \Rightarrow(3) \Rightarrow(4)$. For (3) $\Rightarrow(4)$ observe that (3) will mean that $E$ can be identified with the $f$-subalgebra $\varphi(E)$ of $\operatorname{Orth}(E)$ ( $\varphi$ is the natural $f$-homomorphism from $E$ into $\operatorname{Orth}(E))$ and that $\varphi(x) \wedge I \in \varphi(F)$ for all $x \in F_{+}$; hence it suffices to show that (2) $\Rightarrow(4)$, which follows from

$$
\left(x-n^{-1} e\right)^{+}(e \wedge n x)=\left(x-n^{-1} e\right)^{+} \quad\left(x \in F_{+}, n=1,2, \ldots\right) .
$$

Observe also that any quasi-unital Archimedean $f$-algebra $E$ is nowhere trivial. Let indeed $x \in E$ such that $x^{2}=0$; if $x \neq 0$ there would exist $0<x^{\prime} \leqslant|x|$ with $x^{\prime} y=x^{\prime}$ for some $y \in E_{+}$; it follows easily from $x^{\prime 2}=0$ that $x^{\prime}=x^{\prime} y=0$, and this contradiction establishes the result.

EXAMPLES. (1) An example of a super quasi-unital Archimedean f-algebra without unit is given by all continuous functions with compact support on a locally compact non compact topological space.

(2) A quasi-unital Archimedean f-algebra which is not super quasi-unital. Let $K_{j}=[1,2]$ for all $j$ in some non countable set $J$ and $\Omega=\Sigma K_{j}$ (topological sum); for $u \in \mathcal{C}(\Omega)$ denote by $u_{j}$ its restriction to $K_{j}$. Let $\mathscr{P}$ be the Riesz subspace of $\mathcal{C}$ $[1,2]$ generated by polynomials in $\mathbf{R}[x]$ and define $x \mathscr{P}=\{x f ; f \in \mathscr{P}\} ; \mathscr{P}$ and $x \mathscr{P}$ are $f$-algebras for the usual product. Let also $E$ be the $f$-subalgebra of $\mathcal{C}(\Omega)$ consisting of all $u$ such that $u_{j} \in \mathscr{P}$ for all $j \in J$ and $\left\{j \in J ; u_{j} \notin x \mathscr{P}\right\}$ is at most countable. Observing that $f g \neq f$ for all $f, g \in x \mathscr{P}$, it is easily seen that $E$ is quasi-unital but not super quasi-unital.

(3) If $E=x \mathscr{P}$, then $E$ is not quasi-unital for the usual product. But if for $f=x h, g=x k(h, k \in \mathscr{P})$ we define $f * g=x h k$ (usual product), then $E$ equipped with the product "*" is an $f$-algebra with the polynomial " $x$ " as unit.

The next two propositions provide other examples of super quasi-unital $f$-algebras.

Proposition 2.1. Let $E$ be a nowhere trivial Archimedean f-algebra. If $Z(E)$ is uniformly complete (this occurs in particular if $E$ is uniformly complete), then $E$ is super quasi-unital.

Proof. Fix $x \in E_{+}$, define $T \in \operatorname{Orth}(E)$ by $T y=x y$ and, for $n=1,2, \ldots$, define also $T_{n}=\left(T \vee n^{-1} I\right) \wedge n I$, and $x_{n}=\left(x-n^{-1} x^{2}\right)^{+} \wedge n\left(x^{2}-n^{-1} x\right)^{+}$. Since $E$ is nowhere trivial, we have $b\left(x^{2}\right)=b(x)$; hence $x=\sup x_{n}$. It follows easily from $\left(n^{-1} I-T\right)^{+} x_{n}=(T-n I)^{+} x_{n}=0$ that $n^{-1} x_{n} \leqslant x x_{n} \leqslant n x_{n}$ and, so, $T_{n} x_{n}=x x_{n}$. Now the uniform completeness of $Z(E)$ and $n^{-1} I \leqslant T_{n} \leqslant n I$ imply that $S_{n} T_{n}=I$ for some $S_{n} \in Z(E)$ (1.7). Defining $y_{n}=S_{n} x$, it remains to observe that $x_{n}=S_{n}\left(T_{n} x_{n}\right)=S_{n}\left(x x_{n}\right)=x_{n} y_{n}$. 
Proposition 2.2. Any uniformly complete Archimedean Riesz space E contains an order dense ideal which is a super quasi-unital f-algebra.

Proof. Let $\left(e_{\alpha}\right)$ be a maximal disjoint system in $E_{+}$and let $A$ be the direct sum of all $E_{\alpha}=i\left(e_{\alpha}\right) ; A$ is an order dense ideal in $E$. The Yosida representation of $E_{\alpha}$ as $\mathcal{C}\left(X_{\alpha}\right)$, with $e_{\alpha}=1$, for some compact topological space $X_{\alpha}$, shows that $E_{\alpha}$ can be equipped with a structure of $f$-algebra with unit $e_{\alpha}$. Define $\left(\sum x_{\alpha}\right)\left(\sum y_{\alpha}\right)=$ $\sum x_{\alpha} y_{\alpha}\left(x_{\alpha}, y_{\alpha} \in E_{\alpha} ; x_{\alpha}=y_{\alpha}=0\right.$ for all but a finite number of choices for $\left.\alpha\right)$. The Riesz space $A$ becomes so an $f$-algebra and, for every $x=\sum x_{\alpha} \in A$, there exists $y=\Sigma y_{\alpha} \in A_{+}$such that $x y=x$; it suffices to define $y_{\alpha}=e_{\alpha}$ if $x_{\alpha} \neq 0$, and $y_{\alpha}=0$ if $x_{\alpha}=0$.

The next lemma is needed in order to obtain the main result of this paper.

LEMma 2.3. If $F$ is a (super) quasi-unital Riesz subspace of the Archimedean $f$-algebra $E$, then every ideal $D$ in $F$ is (super) quasi-unital.

Proof. Define $M=\left\{x \in F ; x y=x\right.$ for some $\left.y \in F_{+}\right\}$and $N=\{x \in D$; $x y=x$ for some $\left.y \in D_{+}\right\}$. By hypothesis $i_{F}(M)$ is (super) order dense in $F$, hence $i_{F}(M) \cap D$ is (super) order dense in $D$, and we have to prove that $i_{D}(N)$ is (super) order dense in $D$. It is thus sufficient to show that $i_{D}(N)$ is super order dense in $i_{F}(M) \cap D$; but every member of $i_{F}(M) \cap D_{+}$is a finite sum of members of $M \cap D_{+}$and, so, we have only to show that every $x \in M \cap D_{+}$is the supremum of some sequence in $i_{D}(N)$. Let $y \in F_{+}$such that $x y=x$ and, $n=1,2, \ldots$, define

$$
x_{n}=\left(x-n^{-1} y\right)^{+}, \quad y_{n}=y \wedge n x,
$$

and observe that $x_{n}, y_{n} \in D, 0 \leqslant x_{n} \uparrow x$, and that

$$
x_{n} y_{n}=n x_{n}\left(n^{-1} y-\left(x-n^{-1} y\right)^{-}\right)=x_{n} y=x_{n} \text {. }
$$

Hence $x_{n} \in N$ and the proof is complete.

THEOREM 2.4. Let $E$ be an Archimedean f-algebra and $F$ a quasi-unital Riesz subspace of $E$. Then every $T \in \operatorname{Orth}^{\infty}(F)$ can be extended to $\tilde{T} \in \operatorname{Orth}^{\infty}(E, b(F))$ in the sense that $T=\tilde{T}$ on some order dense ideal of $F$.

Proof. The proof is divided into two parts: given $T \in \operatorname{Orth}^{\circ}(F)$, we shall define $\tilde{T} \in \operatorname{Orth}^{\infty}(E)$ in the first part and, in the second one, we shall verify that $T=\tilde{T}$ on some order dense ideal of $F$.

(1) Let $T \in \operatorname{Orth}^{\infty}(F)$; by $1.1, D=\left\{x \in D_{T} ; T x \in i_{F}(x)\right\}$ is an order dense ideal of $F$. Define $K=\left\{x \in E ; x y=x\right.$ for some $\left.y \in D_{+}\right\}$and $J=i_{E}(K)$. For 
any $x \in J_{+}$write $x=\sum_{i=1}^{n} x_{i}\left(0 \leqslant x_{i} \in K\right)$, choose $y_{i} \in D_{+}$such that $x_{i} y_{i}=x_{i}$ $(1 \leqslant i \leqslant n)$, and define $\tilde{T} x=\sum_{i=1}^{n} x_{i} T y_{i}$. In order to verify that $\tilde{T}$ is well-defined, let $u \in E$ and $v, v^{\prime} \in D_{+}$such that $u v=u v^{\prime}=u$; then $u T v=u T v^{\prime}$ since, for some $\lambda \in \mathbf{R}_{+}$, we have:

$$
\left|u T\left(v-v^{\prime}\right)\right|=|u| \cdot\left|T\left(v-v^{\prime}\right)\right| \leqslant|u| \cdot \lambda\left|v-v^{\prime}\right|=\lambda\left|u\left(v-v^{\prime}\right)\right|=0 .
$$

On the other hand, if $x=\sum_{j=1}^{p} x_{j}^{\prime}$ with $x_{j}^{\prime} \in E_{+}$and $x_{j}^{\prime} y_{j}^{\prime}=x_{j}^{\prime}$ for some $y_{j}^{\prime} \in D_{+}$ $(1 \leqslant j \leqslant p$ ), then it follows from the Riesz decomposition property that there exists a sequence $\left(x_{i j}\right)$ in $E_{+}$such that $x_{i}=\sum_{j=1}^{p} x_{i j}(1 \leqslant i \leqslant n)$, and $x_{j}^{\prime}=\sum_{i=1}^{n} x_{i j}$ $(1 \leqslant j \leqslant p)$. Since $x_{i j} y_{i}=x_{i j} y_{j}^{\prime}=x_{i j}$, it follows that

$$
\sum_{i} x_{i} T y_{i}=\sum_{i, j} x_{i j} T y_{i}=\sum_{i, j} x_{i j} T y_{j}^{\prime}=\sum_{j} x_{j}^{\prime} T y_{j}^{\prime} .
$$

Hence $\tilde{T}: J_{+} \rightarrow J$ is well-defined, additive, positively homogeneous, and $\tilde{T} x \in$ $i_{E}(x)$ for all $x \in J_{+}$. Extending $\tilde{T}$ by linearity to $\tilde{T} \in \operatorname{Orth}(J)$ and setting $\tilde{T}=0$ on $J^{d}$, we obtain a member $\tilde{T}: J \oplus J^{d} \rightarrow E$ of $\operatorname{Orth}^{\infty}\left(E, b_{E}(F)\right.$ ) (since $\tilde{T}=0$ on $\left.J \cap F^{d}\right)$.

(2) The lemma 2.3 shows that $D$ is quasi-unital, that is, $i_{D}(D \cap K)=i_{F}(D \cap$ $K)$ is order dense in $D$, hence in $F$. It remains to see that $T=\tilde{T}$ on $i_{F}(D \cap K)$; since $i_{F}(D \cap K)$ is linearly generated by $D_{+} \cap K$, it suffices to verify that $T=\tilde{T}$ on $D_{+} \cap K$.

To this end fix $x \in D_{+} \cap K$ and choose $y \in D_{+}$such that $x y=x$; then $\tilde{T} x=x T y$ by definition of $\tilde{T}$. since $T y \in i_{F}(y)$, there is some $\lambda \in \mathbf{R}_{+}$such that $|T y| \leqslant \lambda y$ and, by 1.4 , we also have $|T u| \leqslant \lambda u$ for all $u \geqslant 0$ in $b_{F}(y) \cap D=b_{D}(y)$. Hence the restriction of $T$ to $b_{D}(y)$ is in $Z\left(b_{D}(y)\right)$ and, by 1.2 , this restriction can be extended to some $\hat{T} \in Z(\hat{E})$, where $\hat{E}$ is the Dedekind completion of $E$. Note that $\hat{T} x=T x$ since $x=x y \in b_{E}(y) \cap D \subseteq b_{D}(y)$. On the other hand, if $S \in$ $\operatorname{Orth}(E)$ is defined by $S u=x u$ for all $u \in E$, then $S$ can be extended by order continuity to $\hat{S} \in \operatorname{Orth}(\hat{E})$. We finally obtain

$$
\tilde{T} x=x T y=\hat{S}(\hat{T} y)=\hat{T}(\hat{S} y)=\hat{T}(x y)=\hat{T} x=T x,
$$

and the proof is complete.

The next result is an improvement of Theorem 2.4 .

THEOREM 2.5. Let $E$ be an Archimedean Riesz space, $F$ a Riesz subspace of $E$ and assume there exists an ideal $A$ of $E$ such that:

(1) $F \cap A$ is order dense in $F$;

(2) $A$ is an f-algebra and $F \cap A$ is quasi-unital.

Then every $T \in \operatorname{Orth}^{\infty}(F)$ can be extended to $\tilde{T} \in \operatorname{Orth}^{\infty}(E, B)$, with $B=b(F)$, in the sense that $T=\tilde{T}$ on some order dense ideal in $F$. If $F$ is order dense in $B$, then $\tilde{T}=\psi(T)$ is unique and $\psi$ is an f-isomorphism from $\operatorname{Orth}^{\infty}(F)$ into $\operatorname{Orth}^{\infty}(E, B)$; moreover $T=\tilde{T}$ on $D_{T}^{m} \cap D_{\tilde{T}}^{m}$. 
Proof. Let $T \in \operatorname{Orth}^{\infty}(F)$ and define $D=\left\{x \in D_{T} ; T x \in i_{F}(x)\right\}$. Since $D$ is an order dense ideal in $F(1.1)$, the restriction of $T$ to $D \cap A$ is in $\operatorname{Orth}^{\infty}(F \cap A)$ (actually, $\left.\operatorname{Orth}^{\infty}(F \cap A)=\operatorname{Orth}^{\infty}(F)\right)$. Applying 2.4 to the quasi-unital Riesz subspace $F \cap A$ of the $f$-algebra $A$, we get $\tilde{T} \in \operatorname{Orth}^{\infty}\left(A, b_{A}(F)\right.$ ), with domain $\tilde{D}$, and an order dense ideal $L$ of $F \cap A$ such that $T=\tilde{T}$ on $L$. But $L$ is also an order dense ideal in $F$ and, moreover, $\tilde{T}$ can be extended to a member $\tilde{T}: \tilde{D} \oplus A^{d} \rightarrow E$ of $\operatorname{Orth}^{\infty}(E, B)$ by defining $\tilde{T}=0$ on $A^{d}$. The results in the special case when $F$ is order dense in $B$ are easy consequences of the order continuity of extended orthomorphisms.

EXAMPLES. (1) Considering the situation of 2.5 we show that if $E=b(F)$, but $F$ is not order dense in $E$, it can happen that $T \in \operatorname{Orth}^{\infty}(F)$ and one of its "extension" $\tilde{T} \in \operatorname{Orth}^{\infty}(E)$ do not coincide on $D_{T}^{m} \cap D_{\tilde{T}}^{m}$ (even if $D_{T}^{m} \subseteq D_{T}^{m}$ ). Take $E=\mathscr{F}[0,1]$ (all the real valued functions on $[0,1]), F=\mathcal{C}[0,1]$, and define $T \in \operatorname{Orth}^{\infty}(F)$ by $(T f)(x)=f(x) / x$; then $D_{T}^{m}=\left\{f \in F ; \lim _{x \rightarrow 0} f(x) / x=0\right\}$. Define also $\tilde{T} \in \operatorname{Orth}(E)$ by $\tilde{T} f=g f$ for all $f \in E$, where $g \in E$ is defined by $g(x)=1 / x(x \neq 0,1), g(0)=g(1)=0$. Then $\tilde{T}$ is an "extension" of $T$ since $T=\tilde{T}$ on the order dense ideal $D=\left\{f \in D_{T}^{m} ; f(1)=0\right\}$ of $F$, but clearly $T f \neq \tilde{T} f$ for all $f \in D_{T}^{m} \backslash D$. Observe also that $E, F$ are $f$-algebras with unit and are uniformly complete ( $E$ is even laterally and Dedekind complete).

(2) Even if $F$ is order dense in $E$, it is not necessarily true that $T \in \operatorname{Orth}^{\infty}(F)$ and its unique extension $\tilde{T} \in \operatorname{Orth}^{\infty}(E)$ satisfy $D_{T}^{m} \subseteq D_{\tilde{T}}^{m}$. take $E=\mathcal{C}[0,1]$ and consider the order dense Riesz subspace

$$
F=\{f \in E ; f \text { is polynomial on some neighborhood of } 0\} .
$$

Defining $T \in \operatorname{Orth}^{\infty}(F)$ by $(T f)(x)=f(x) / x$, we have

$$
D_{T}^{m}=\left\{f \in F ; \lim _{x \rightarrow 0} f(x) / x \text { exists and is finite }\right\}=\{f \in F ; f(0)=0\} .
$$

The unique extension $\tilde{T} \in \operatorname{Orth}^{\infty}(E)$ is obviously defined by $(\tilde{T} f)(x)=f(x) / x$, and $D_{\tilde{T}}^{m}=\left\{f \in E ; \lim _{x \rightarrow 0} f(x) / x=0\right\}$. It is clear that $D_{T}^{m} \nsubseteq D_{\tilde{T}}^{m}$, although $E, F$ are $f$-algebras with unit and $E$ is uniformly complete.

(3) We finally show that 2.5 may fail without any special hypothesis on $E$ of $F$; our example is inspired from [16; example (a)]. Let $F$ be the Riesz subspace of $\mathcal{C}[0,1]$ generated by polynomials and $E$ the one generated by $F$ and $\exp (x)$; then $F$ is an order (and uniformly) dense Riesz subspace of $E$, containing 1. Moreover $F$ is an $f$-algebra, $Z(F)=F$ (operating by product), and $Z(E)=\{\lambda I ; \lambda \in \mathbf{R}\}$. Let $C$ be the collection of all constant functions on $[0,1]$. Then with the notations of $[3 ; 4.3]$, we have $\operatorname{Orth}^{\infty}(F)=L(F, \mathscr{Q},[0,1])$ and $\operatorname{Orth}^{\infty}(E)=L(C, \mathscr{D},[0,1])$. More precisely, $\mathscr{D}$ is the collection of all dense open subsets of $[0,1]$ and, if $G$ is a Riesz subspace of $\mathcal{C}[0,1]$, then $L(G, \mathcal{D},[0,1])$ is the collection of all real valued 
functions $f$ which are locally in $G$ with respect to $\mathscr{D}$, in the sense that: (a) $f$ is defined on some $U_{f} \in \mathscr{Q}$; (b) for every $t \in U_{f}$ there exists a neighborhood $V$ of $t$ and $g \in G$ such that $f=g$ on $U_{f} \cap V$.

The next result shows how powerful Theorem 2.5 is

COROllaRy 2.6. Let $E$ be a uniformly complete Archimedean Riesz space and $F$ any Riesz subspace of $E$. Then every $T \in \operatorname{Orth}^{\infty}(F)$ can be extended to $\tilde{T} \in$ $\operatorname{Orth}^{\infty}(E, B)$, with $B=b(F)$, in the sense that $T=\tilde{T}$ on some order dense ideal of $F$. If $F$ is order dense in $B$, then $\tilde{T}=\psi(T)$ is unique and $\psi$ is an f-isomorphism from $\operatorname{Orth}^{\infty}(F)$ into $\operatorname{Orth}^{\infty}(E, B)$; moreover $T=\tilde{T}$ on $D_{T}^{m} \cap D_{\tilde{T}}^{m}$.

Proof. In order to obtain an ideal $A$ of $E$ satisfying conditions (1) and (2) of 2.5 , consider a maximal disjoint system $\left(e_{\alpha}\right)$ in $F_{+}$and let $A$ be the direct sum of all $E_{\alpha}=i_{E}\left(e_{\alpha}\right)$. Continue as in the proof of 2.2 .

REMARKs. (1) Even if $F$ is order dense in $E$, it is not necessarily true that $D_{T}^{m} \subseteq D_{\tilde{T}}^{m}$ (see example 2 after 2.5). However if $E$ is a Dedekind ( $\sigma$-) complete Riesz space and $F$ a (super) order dense Riesz subspace of $E$, then every $T \in \operatorname{Orth}^{\infty}(F)$ can be extended by order continuity to (a unique) $\tilde{T} \in \operatorname{Orth}^{\infty}(E)$ such that $D_{T}^{m} \subseteq D_{\tilde{T}}^{m}$.

(2) In a recent unpublished paper [13], B. de Pagter has obtained, with a completely different proof, the following result, which is a special case of 2.6 : If $E, F$ are uniformly complete Archimedean Riesz spaces such that $F$ is an order dense Riesz subspace of $E$, then any $T \in \operatorname{Orth}^{\infty}(F)$ has an extension $\tilde{T} \in$ $\operatorname{Orth}^{\infty}(E)$.

Before starting the next corollary, we observe that the richness of $Z(E)$ or $\operatorname{Orth}(E)$ are equivalent properties (since the identity $I$ on $E$ is a weak order unit in $\left.\operatorname{Orth}^{\infty}(E)\right)$.

COROllaRy 2.7. If the Archimedean Riesz space $E$ has a rich center, then there exists an f-isomorphism $\psi$ from $\operatorname{Orth}^{\infty}(\operatorname{Orth}(E))$ into $\operatorname{Orth}^{\infty}(E)$ such that $U(T)=$ $\psi(U) \cdot T$ for all $U \in \operatorname{Orth}^{\infty}(\operatorname{Orth}(E))$ and all $T \in D_{U}^{m}$.

Proof. Let $U \in \operatorname{Orth}^{\infty}(\operatorname{Orth}(E))$; since, by hypothesis, $\operatorname{Orth}(E)$ is order dense in $\operatorname{Orth}^{\infty}(E)$, it follows from 2.4 that there exists $\tilde{U} \in \operatorname{Orth}^{\infty}\left(\operatorname{Orth}^{\infty}(E)\right)$ such that $U=\tilde{U}$ on $D_{U}^{m} \cap D_{\tilde{U}}^{m}$. But, by $1.6, \tilde{U}$ is in $\operatorname{Orth}\left(\operatorname{Orth}^{\infty}(E)\right)$ and there exists $S \in \operatorname{Orth}^{\infty}(E)$ such that $\tilde{U}(T)=S T$ for all $T \in \operatorname{Orth}^{\infty}(E)$. In particular $U(T)=$ $S T$ for all $T \in D_{U}^{m}$ and, since $D_{U}^{m}$ is order dense in $\operatorname{Orth}^{\infty}(E)$, such an $S$ is unique. It remains to define $\psi(U)=S$. 
Given an Archimedean Riesz space $E$ with a rich center, we shall say that $\operatorname{Orth}^{\infty}(\operatorname{Orth}(E))=\operatorname{Orth}^{\infty}(E)$ if the $f$-isomorphism $\psi$, defined in 2.7 , is onto or, in other words, if for every $S \in \operatorname{Orth}^{\infty}(E)$ there exists an order dense ideal $\mathbb{Q}$ in $\operatorname{Orth}(E)$ such that $S T \in \operatorname{Orth}(E)$ for all $T \in Q$.

We conclude this section by giving conditions to have $\operatorname{Orth}^{\infty}(\operatorname{Orth}(E))=$ $\operatorname{Orth}^{\infty}(E)$. For this the next two lemmas are needed.

Lemma 2.8. An Archimedean f-algebra $E$ is quasi-unital if and only if $E$ is nowhere trivial and contains an order dense ideal of $\operatorname{Orth}(E)$.

Proof. If $E$ is quasi-unital, we already know that $E$ is nowhere trivial and, so, the natural $f$-homomorphism $\varphi$ from $E$ into $\operatorname{Orth}(E)$ is one-to-one; hence $\varphi(E)$ is rich, that is, order dense in $\operatorname{Orth}^{\infty}(E)$, since $0 \neq \varphi(x) \in \operatorname{Orth}(E, b(x))$ for all $x \neq 0$ in $E$. Define $M=\left\{x \in E ; x y=x\right.$ for some $\left.y \in E_{+}\right\}$. Then $\varphi\left(i_{E}(M)\right)$ is order dense in $\operatorname{Orth}(E)$ since $E$ is quasi-unital and, moreover, $\varphi\left(i_{E}(M)\right)$ is an ideal in $\operatorname{Orth}(E)$ : it suffices to observe that if $0 \leqslant T \leqslant \varphi(x)$, with $T \in \operatorname{Orth}(E)$, $x \in E$, and $x y=x$ for some $y \in E_{+}$, then $T=\varphi(T y)$. The converse result follows from 2.3 .

Given an Archimedean Riesz space $E$ and $T \in \operatorname{Orth}^{\infty}(E)$, define

$$
A_{T}=\left\{x \in D_{T}^{m} ; T x \in i(x)\right\} ;
$$

by 1.1, $A_{T}$ is an order dense ideal in $E$. A Riesz space is said to be order Cauchy complete if every order Cauchy sequence is order convergent; order Cauchy completeness is weaker than Dedekind $\sigma$-completeness and stronger than uniform completeness.

Lemma 2.9. For an Archimedean Riesz space E, the following statements are equivalent:

(1) $E$ is order Cauchy complete;

(2) $E$ is uniformly complete and $b(x) \subseteq D_{T}^{m}$ for all $T \in \operatorname{Orth}^{\infty}(E)$ and all $x \in A_{T}$.

Proof. (1) $\Rightarrow(2)$. Observe first that if $x \in E$, then every $T \in Z(i(x))$ can be extended to $S \in Z(b(x))$. Indeed, assuming that $0 \leqslant T \leqslant \lambda I$ for some $\lambda \in \mathbf{R}_{+}$ and considering $0 \leqslant y \in b(x)$, we can define $S y=\sup _{n} T y_{n}$, where $\left(y_{n}\right)$ is any sequence in $i(x)$ such that $0 \leqslant y_{n} \uparrow y$; the supremum exists because it follows from

$$
0 \leqslant T y_{n+p}-T y_{n} \leqslant \lambda\left(y_{n+p}-y_{n}\right) \leqslant \lambda\left(y-y_{n}\right) \downarrow_{n} 0
$$

that $\left(T y_{n}\right)$ is an order Cauchy sequence in $E$. 
Now let $T \in \operatorname{Orth}^{\infty}(E)$ and $x \in A_{T}$; then the restriction of $T$ to $i(x)$ is in $Z(i(x))$ and, hence, this restriction can be extended to $S \in Z(b(x))$. The mapping

$$
D_{T}^{m}+b(x) \rightarrow E:(y+z) \mapsto T y+S z
$$

is well-defined (because $T=S$ on $D_{T}^{m} \cap b(x)$ ), is an extension of $T$ and an extended orthomorphism on $E$; hence $b(x) \subseteq D_{T}^{m}$.

$(2) \Rightarrow(1)$. By $[3 ; 3.11]$ and uniform completeness of $E$, it suffices to see that, given $x \in E_{+}$, every $T \in Z(i(x))$ can be extended to $S \in Z(b(x))$. But the linear mapping $R: i(x) \oplus i(x)^{d} \rightarrow E$, defined by $R=T$ on $i(x)$ and $R=0$ on $i(x)^{d}$, is in $\operatorname{Orth}^{\infty}(E)$ and $x \in A_{R}$. Hence $b(x) \subseteq D_{R}^{m}$ and the restriction $S$ of $R$ to $b(x)$ is in $Z(b(x))$ and is an extension of $T$.

A Riesz space is said to be disjointly complete if every positive order bounded disjoint subset has a supremum.

THEOREM 2.10. Let $E$ be an Archimedean Riesz space and assume that $E$ has an order dense ideal $F$ such that $Z(E)=Z(F)$ (that is, every $T \in Z(F)$ can be extended to $T \in Z(E)$ ) and such that one of the following hypothesis holds:

(1) $F$ is disjointly complete;

(2) $F$ is a quasi-unital f-algebra;

(3) $Z(F)$ is ultrarich;

(4) $Z(F)$ is rich and $F$ is order Cauchy complete.

Then $Z(E)$ is rich and $\operatorname{Orth}^{\infty}(\operatorname{Orth}(E))=\operatorname{Orth}^{\infty}(E)$.

Proof. Since $F$ is an order dense ideal in $E$, it follows from 1.1 that $\operatorname{Orth}^{\infty}(E)=\operatorname{Orth}^{\infty}(F)$. Since $Z(E)$ is a (super) order dense ideal in $\operatorname{Orth}(E)$, and similarly for $F$, we have also:

$$
\operatorname{Orth}^{\infty}(\operatorname{Orth}(E))=\operatorname{Orth}^{\infty}(Z(E))=\operatorname{Orth}^{\infty}(Z(F))=\operatorname{Orth}^{\infty}(\operatorname{Orth}(F)) .
$$

Observing moreover that $Z(E)$ is rich if and only if $Z(F)$ is rich, it is thus sufficient to verify that $Z(F)$ is rich and that $\operatorname{Orth}^{\infty}(\operatorname{Orth}(F))=\operatorname{Orth}^{\infty}(F)$. Recall that this last equality will mean that, for every $T \in \operatorname{Orth}^{\infty}(F)$, there exists some order dense ideal $\mathbb{Q}$ in $\operatorname{Orth}(F)$ such that $T S \in \operatorname{Orth}(F)$ for all $S \in \mathbb{Q}$.

Let $T \in \operatorname{Orth}^{\infty}(F)$. If (1) is true then, by [3;2.8.2], $\operatorname{Orth}(F)$ is a (super) order dense ideal in $\operatorname{Orth}^{\infty}(F)$, and the result follows from 1.1 applied to $U \in$ $\operatorname{Orth}\left(\operatorname{Orth}^{\infty}(F)\right)$ defined by $U(S)=T S$. If (2) is true then $Z(F)$ is rich and, by 2.8, $F$ contains an order dense ideal of $\operatorname{Orth}(F)$; hence the result follows from 1.1 applied to $T$. If (3) holds, then we can take $Q=Z\left(F, D_{T}^{m}\right)$ since $Z\left(F, D_{T}^{m}\right)$ is obviously ultrarich. If (4) holds we can also take $\mathscr{Q}=Z\left(F, D_{T}^{m}\right)$ since, by 2.9 and richness of $Z(F), Z\left(F, D_{T}^{m}\right)$ is rich. 
REMARKS. (1) We have no counterexample showing that the richness of $E$ is not sufficient to insure that $\operatorname{Orth}^{\infty}(\operatorname{Orth}(E))=\operatorname{Orth}^{\infty}(E)$.

(2) Any Dedekind $\sigma$-complete Riesz space $F$ satisfies (4) of 2.10 . By [3; 3.10], any order Cauchy complete Archimedean Riesz space $F$ with a weak order unit satisfies (4). If $K$ is a compact topological space, then $\mathcal{C}(K)$ has an ultrarich center ( $f$-isomorphic to $\mathcal{C}(K)$ ); hence if $E$ is a Banach lattice with a quasi-interior point $u$, it follows from the Yosida representation theorem that $F=i(u)$ satisfies (2) and (3) of 2.10 and, moreover, $Z(E)=Z(F)$ since every $T \in Z(F)$ is norm continuous. More generally, if $E$ is a Banach lattice with a representation space in the sense of [14; III, 5.4], then the same argument works.

\section{Inversion in $\operatorname{Orth}^{\infty}(E)$}

We start with a few comments about our hypothesis in the results of this section. Considering the following conditions on an Archimedean Riesz space $E$,

(1) $E$ is uniformly complete,

(2) $Z(i(x))$ is uniformly complete for every $x \in E_{+}$,

(3) $Z(E)$ is uniformly complete,

it is easy to verify that $(1) \Rightarrow(2) \Rightarrow(3)$, but $(2) \Rightarrow(1)$ and $(3) \Rightarrow(2)$ are not true as shown in the next examples.

EXAmples. (1) Let $E=A_{m}[0,1]$ be the Riesz space of all continuous piecewise linear functions on $[0,1]$. Then for every $f \in E_{+}, Z(i(f))$ is finite dimensional and, hence, is uniformly complete, but $E$ is obviously not uniformly complete.

(2) Let $E$ be the Riesz space of all continuous functions if on $\mathbf{R}_{+}$such that: (a) for every integer $n, f$ is piecewise linear on $[n, n+1]$; (b) there exists $m$ (depending on $f$ ) such that $f(x+1)=f(x)$ for all $x \geqslant m$. It is easily seen that $Z(E)$ is one dimensional and thus uniformly complete. Defining $g \in E_{+}$by $g(x+1)=g(x)$ for all $x$ and $g(t)=t$ if $t \in\left[0,2^{-1}\right],=1-t$ if $t \in\left[2^{-1}, 1\right]$, it is not difficult to verify that $Z(i(g))$ is not uniformly complete.

LEMma 3.1. Let $E$ be any Archimedean Riesz space and $T \in \operatorname{Orth}^{\infty}(E)$. Then $|T|$ is a weak order unit in $\operatorname{Orth}^{\infty}(E)$ if and only if $B_{T}=E$. It is true, in particular, if $T$ has an inverse in $\operatorname{Orth}^{\infty}(E)$.

Proof. $\left\{T \mid\right.$ is a weak order unit in $\operatorname{Orth}^{\infty}(E)$ if and only if $I=\sup _{n} I \wedge n|T|$, that is, $x=\sup _{n} x \wedge n|T x|$ for all $0 \leqslant x \in D_{T}$ (1.5). That will mean that $x \in$ $b(T x)$ fr all $0 \leqslant x \in D_{T}$ and, by 1.3 , this is also equivalent to $B_{T}=E$. Since $B_{S T} \subseteq B_{T}$ for any $S, T \in \operatorname{Orth}^{\infty}(E)$, the last assertion follows. 
LEMMA 3.2. Let $E$ be an Archimedean Riesz space such that $Z(i(x))$ is uniformly complete for all $x \in E_{+}$. Let also $T \in \operatorname{Orth}(E)$ and assume that for every $x \in E_{+}$ there exist $0<\lambda \leqslant \mu$ in $\mathbf{R}$ such that $\lambda x \leqslant|T x| \leqslant \mu x$. Then $T$ has an inverse in $\operatorname{Orth}(E)$.

Proof. If $x \in E$ and $\lambda|x| \leqslant|T x| \leqslant \mu|x|$ for some $0<\lambda \leqslant \mu$, then $\lambda|y| \leqslant$ $|T y| \leqslant \mu|y|$ holds for all $y \in i(x)(1.4)$ and, by 1.7, the restriction of $T$ to $i(x)$ has an inverse $S_{x}$ in $Z(i(x))$. If $x, y \in E$ and $x \in i(y)$, then $S_{x} T x=x=S_{y} T x$ and, since $x \in i(T x)$, it follows that $S_{x} x=S_{y} x$. Hence $S: E \rightarrow E$, defined by $S x=S_{x} x$, is a member of $\operatorname{Orth}(E)$ such that $S T=I$.

For every band $B$ in an Archimedean Riesz space $E$, we define the extended orthomorphism $\pi_{B}: B \oplus B^{d} \rightarrow E$ by $\pi_{B}(x+y)=x$ for all $x \in B$ and all $y \in B^{d}$; we shall write $\pi_{B}^{d}$ instead of $\pi_{B^{d}}$. It is easily seen that $B \oplus B^{d}$ and $B$ are respectively the maximal domain and the support of $\pi_{B}$.

THEOREM 3.3. Let $E$ be an Archimedean Riesz space such that $Z(i(x))$ is uniformly complete for all $x \in E_{+}$. Then for every $T \in \operatorname{Orth}^{\infty}(E)$ there exists $S \in \operatorname{Orth}^{\infty}(E)$ such that $S T=\pi_{B_{T}}$.

Proof. Let $T \in \operatorname{Orth}^{\infty}(E)$, define $J=\left\{x \in D_{T} ; \lambda|x| \leqslant|T x| \leqslant \mu|x|\right.$ for some $0<\lambda \leqslant \mu\}$ and observe that $J$ is an ideal in the band $B_{T}$ of $E$. Given $0 \leqslant x \in B_{T}$ $\cap D_{T}$, we have $b(T x)=b(x)(1.3)$, and so $x=\sup x_{n}$, where

$$
x_{n}=\left(x-n^{-1}|T x|\right)^{+} \wedge n\left(|T x|-n^{-1} x\right)^{+} \quad(n=1,2, \ldots),
$$

is in $J$, since $n^{-1} x_{n} \leqslant\left|T x_{n}\right| \leqslant n x_{n}$ for all $n$. That shows that $J$ is super order dense in $B_{T} \cap D_{T}$; hence $J$ is order dense in $B_{T}$. By 3.2 the restriction of $T$ to $J$ has an inverse $S$ in $\operatorname{Orth}(J)$ and, extending $S$ to $J \oplus B_{T}^{d}$ by setting $S=0$ on $B_{T}^{d}$, we obtain an $S \in \operatorname{Orth}^{\infty}(E)$ such that $S T=\pi_{B_{r}}$.

REMARKs. (1) If $E$ is any Archimedean Riesz space, then the assertion in 3.3 continues to hold if we only assume that, for all $x \in E_{+}$, every strong order unit in $Z(i(x))$ has an inverse in $\operatorname{Orth}^{\infty}(i(x))$.

(2) The next example shows that some hypothesis on $E$ in needed to insure that every extended orthomorphism is invertible on its support. Let $E$ be the Riesz subspace of $\mathcal{C}[0,1]$ generated by the polynomials. Then $E$ is an $f$-algebra with unit, but the only members of $\left.\operatorname{Orth}^{\infty}(E)=L(E, \mathscr{D})[0,1]\right)$ which are invertible on their support are the functions in $L(C, \mathscr{D},[0,1])$, where $C$ is the collection of all constant functions (see remark 3 after 2.5 ). 
The next corollary follows immediately from 3.1 and 3.3.

CoRollary 3.4. If $E$ is as in 3.3, then $T \in \operatorname{Orth}^{\infty}(E)$ has an inverse in $\operatorname{Orth}^{\infty}(E)$ if and only if $|T|$ is a weak order unit in $\operatorname{Orth}^{\infty}(E)$.

In [13] it is shown (with a rather technical proof) that if $E$ is a uniformly complete Archimedean Riesz space, then the algebra $\operatorname{Orth}^{\infty}(E)$ is von Neumann regular, that is, for every $T \in \operatorname{Orth}^{\infty}(E)$ there exists $S \in \operatorname{Orth}^{\infty}(E)$ such that $T=S T^{2}$. As a corollary it is also obtained that, when $E$ is uniformly complete, every weak order unit in $\operatorname{Orth}^{\infty}(E)$ is invertible. To conclude this section we remark in the next corollary that the von Neumann regularity of $\operatorname{Orth}^{\infty}(E)$ follows immediately from 3.3.

COROllary 3.5. If $E$ is as in 3.3 , then $\operatorname{Orth}^{\infty}(E)$ is von Neumann regular.

Proof. If $S, T \in \operatorname{Orth}^{\infty}(E)$ satisfy $S T=\pi_{B_{T}}$, then $S T^{2}=\pi_{B_{T}} T=T$.

\section{Applications to $\operatorname{Orth}^{\circ}(E)$}

A o-orthomorphism $T$ on an Archimedean Riesz space $E$ is an extended orthomorphism that can be defined on a super order dense ideal of $E$ or, in other words, such that $D_{T}^{m}$ is super order dense in $E$; the set $\operatorname{Orth}^{\sigma}(E)$ of all $\sigma$-orthomorphisms on $E$ has been introduced and studied in [3]. When we shall consider a possible domain $D_{T}$ for a $\sigma$-orthomorphism $T$, it will be always supposed that $D_{T}$ is super order dense. Using 1.1, it is easily seen that $\operatorname{Orth}^{\sigma}(E)$ is an $f$-subalgebra of $\operatorname{Orth}^{\infty}(E)$; since moreover $I \in \operatorname{Orth}^{\circ}(E)$, it follows that $\operatorname{Orth}\left(\operatorname{Orth}^{\circ}(E)\right)=$ $\operatorname{Orth}^{\sigma}(E)$, the members of $\operatorname{Orth}^{\sigma}(E)$ operating by product on $\operatorname{Orth}^{\circ}(E)$.

Note that $\sigma$-orthomorphisms are useful to characterize the universal $\sigma$-completion (see [3]) and the lateral o-completion (see [4]) of many almost Dedekind $\sigma$-complete Riesz spaces (that is, Riesz spaces which are Riesz isomorphic to a super order dense Riesz subspace of some Dedekind $\sigma$-complete Riesz space).

It follows from [3; 3.6, 3.8.1] that if $E$ is an almost Dedekind $\sigma$-complete Riesz space, then $\operatorname{Orth}^{\sigma}\left(\operatorname{Orth}^{\sigma}(E)\right)=\operatorname{Orth}\left(\operatorname{Orth}^{\sigma}(E)\right)$. But the almost Dedekind $\sigma$-completeness of $E$ implies that $\operatorname{Orth}^{\circ}(E)$ is rich $[3 ; 3.4]$ and, as a consequence of 2.4 and the next lemma, we shall show that this richness suffices to insure that $\operatorname{Orth}^{\sigma}\left(\operatorname{Orth}^{\sigma}(E)\right)=\operatorname{Orth}\left(\operatorname{Orth}^{\sigma}(E)\right)$.

LemMa 4.1. Let $E$ be an Archimedean Riesz space such that $\operatorname{Orth}^{\sigma}(E)$ is rich, let $S \in \operatorname{Orth}^{\infty}(E)$, and assume that $S T \in \operatorname{Orth}^{\sigma}(E)$ for all $T$ in some super order dense ideal $\&$ of $\operatorname{Orth}^{\circ}(E)$. Then $S \in \operatorname{Orth}^{\sigma}(E)$. 
Proof. For $T \in \mathbb{Q}$ define the ideal $K(T)=D_{T} \cap D_{S T} \cap \operatorname{Ker}(I-T)$. In order to show that $J=i(\cup\{K(T) ; T \in \mathbb{Q}\})$ is super order dense in $E$, let $\left(T_{n}\right)$ be a sequence in $\mathcal{Q}$ such that $0 \leqslant T_{n} \uparrow I$ in $\operatorname{Orth}^{\circ}(E)$, hence also in $\operatorname{Orth}^{\infty}(E)$ by richness of $\operatorname{Orth}^{a}(E)$; note that $S_{n}=n T_{n} \wedge I$ is in $Q$. Fix $x \in E_{+}$and, for each $n$, choose a sequence $\left(x_{p}^{n}\right)$ in $D_{T_{n}} \cap D_{S S_{n}}$ such that $0 \leqslant x_{p}^{n} \uparrow_{p} x$. It follows from $I=\sup _{n \geq q} T_{n}$, order continuity and 1.5 that $x=\sup \left\{T_{n} x_{p}^{n} ; n \geqslant q\right.$ and $\left.p \geqslant 1\right\}$ $(q=1,2, \ldots)$; hence $x=\sup _{n, p} y_{p}^{n}$ with $y_{p}^{n}=\left(T_{n} x_{p}^{n}-n^{-1} x_{p}^{n}\right)^{+}$. Observe that $y_{p}^{n} \in D_{S_{n}} \cap D_{S S_{n}}$ (since $0 \leqslant T_{n} \leqslant I$ and $D_{T_{n}}=D_{S_{n}}$ ), and also that $y_{p}^{n} \in \operatorname{Ker}(I-$ $S_{n}$ ) since

$$
\left(I-S_{n}\right) y_{p}^{n}=n^{-1}\left(I-n T_{n}\right)^{+}\left(n T_{n}-I\right)^{+} x_{p}^{n}=0 .
$$

Hence $y_{p}^{n} \in K\left(S_{n}\right) \subseteq J$, and $J$ is super order dense in $E$.

It remains to see that $J \subseteq D_{S}^{m}$. But if $T \in \mathbb{Q}$, the mapping

$$
D_{S}^{m}+K(T) \rightarrow E:(x+y) \mapsto S x+S T y
$$

is well-defined (since $S=S T$ on $D_{S}^{m} \cap K(T)$ ) and is obviously an extension of $S$ and an extended orthomorphism on $E$. Hence $K(T) \subseteq D_{S}^{m}$ for all $T \in \mathbb{Q}$, and the proof is complete.

Remark. The same proof shows that if $\operatorname{Orth}(E)$ is rich, $S \in \operatorname{Orth}^{\infty}(E)$, and $S T \in \operatorname{Orth}^{\sigma}(E)$ for all $T$ in some super order dense ideal of $\operatorname{Orth}(E)$, then $S \in \operatorname{Orth}^{\sigma}(E)$. It follows immediately from this fact that the $f$-isomorphism $\psi$ defined in 2.7 satisfies $\psi\left(\operatorname{Orth}^{\circ}(\operatorname{Orth}(E))\right) \subseteq \operatorname{Orth}^{\sigma}(E)$.

THEOREM 4.2. If $E$ is an Archimedean Riesz space such that $\operatorname{Orth}^{\circ}(E)$ is rich, then

$$
\operatorname{Orth}^{\sigma}\left(\operatorname{Orth}^{\sigma}(E)\right)=\operatorname{Orth}\left(\operatorname{Orth}^{\sigma}(E)\right)=\operatorname{Orth}^{\sigma}(E)
$$

Proof. Given $U \in \operatorname{Orth}^{\circ}\left(\operatorname{Orth}^{\circ}(E)\right)$, it suffices to see that $U \in \operatorname{Orth}\left(\operatorname{Orth}^{\circ}(E)\right)$. Since $\operatorname{Orth}^{\sigma}(E)$ is order dense in $\operatorname{Orth}^{\infty}(E)$, it follows from 2.4 and 1.6 that there exists $\tilde{U} \in \operatorname{Orth}\left(\operatorname{Orth}^{\infty}(E)\right)$ such that $U=\tilde{U}$ on $D_{U}^{m}$, and also $S \in \operatorname{Orth}^{\infty}(E)$ such that $\tilde{U}(T)=S T$ for all $T \in \operatorname{Orth}^{\infty}(E)$. Hence $U(T)=S T$ for all $T \in D_{U}^{m}$ and, by $4.1, S$ is in $\operatorname{Orth}^{\sigma}(E)$. Finally, $U=\bar{S}$ where $\bar{S} \in \operatorname{Orth}\left(\operatorname{Orth}^{\sigma}(E)\right)$ is defined by $\bar{S}(T)=S T$.

Remarks. (1) We do not know if the richness of $\operatorname{Orth}^{\sigma}(E)$ is necessary for 4.2 and, even, we have no example of a Riesz space $E$ such that $\operatorname{Orth}^{\sigma}(E)$ is not rich.

(2) It follows from 4.2 and $[3 ; 3.10]$ that if $\operatorname{Orth}^{\sigma}(E)$ is rich, then uniform completeness and order Cauchy completeness are equivalent properties for $\operatorname{Orth}^{\circ}(E)$. 
Let $E$ be an Archimedean Riesz space and $\mathcal{Q}$ a Riesz subspace of $\operatorname{Orth}(E)$. We shall say that $\mathcal{Q}$ is super rich (resp. super ultrarich) if $E$ is countably generated (that is, is the band in itself generated by some sequence) and if, for every $x \in E_{+}$, there exists a sequence $\left(T_{n}\right)$ in $\mathscr{Q}(b(x))$ (resp. in $\mathscr{Q}(i(x))$ ) such that $x=\sup _{n} T_{n} x$. Note that in the examples at the end of Section 1 , the ultrarich center of $E=\mathscr{K}(\Omega)$ is not super rich if $\Omega$ is not $\sigma$-compact, and that $E=L^{p}(\mu)$ has a super rich but not ultrarich center. A Riesz space is said to be disjointly $\sigma$-complete if every positive order bounded disjoint sequence has a supremum. With these definitions we can state the next theorem which is similar to 2.10 .

TheOREM 4.3. Let $E$ be an Archimedean Riesz space and assume that $E$ has a super order sense ideal $F$ such that $Z(E)=Z(F)$ and such that one of the following hypothesis holds:

(1) $F$ is disjointly $\sigma$-complete;

(2) $F$ is a countably generated super quasi-unital f-algebra;

(3) $Z(F)$ is super ultrarich;

(4) $Z(F)$ is super rich and $F$ is order Cauchy complete.

Then $Z(E)$ is rich and $\operatorname{Orth}^{\circ}(\operatorname{Orth}(E))=\operatorname{Orth}^{\sigma}(E)$.

Remark. Any Dedekind $\sigma$-complege Riesz space $F$ satisfies (1) of 4.3. Any order Cauchy complete Archimedean Riesz space $F$ with a weak order unit satisfies (4). If $E$ is a Banach lattice with a quasi-interior point $u$, then $F=i(u)$ satisfies (2) and (3) of 4.3, and $Z(F)=Z(E)$.

Now we shall investigate the invertibility of $\sigma$-orthomorphisms.

TheOREM 4.4. Let $E$ be an Archimedean Riesz space such that $Z(i(x))$ is uniformly complete for all $x \in E_{+}$. If $T \in \operatorname{Orth}^{\circ}(E)$, then there exists $S \in \operatorname{Orth}^{\circ}(E)$ such that $S T=\pi_{B_{T}}$ if and only if $B_{T} \oplus B_{T}^{d}$ is super order dense in $E$.

Proof. Let $T \in \operatorname{Orth}^{\sigma}(E)$ and use the notations of the proof of 3.3. The super order dense ideal $J$ of $B_{T} \cap D_{T}$ is now also super order dense in $B_{T}$ and, so, if $B_{T} \oplus B_{T}^{d}$ is super order dense in $E$, then $J \oplus B_{T}^{d}$ has the same property; hence $S$ : $J \oplus B_{T}^{d} \rightarrow E$ is a $\sigma$-orthomorphism. Conversely, if $S T=\pi_{B_{T}}$ for some $S \in$ $\operatorname{Orth}^{\sigma}(E)$, then $\pi_{B_{T}} \in \operatorname{Orth}^{\sigma}(E)$ and, consequently, the maximal domain $B_{T} \oplus B_{T}^{d}$ of $\pi_{B_{T}}$ is super order dense in $E$.

The next corollary follows immediately from 3.1 and 4.4.

Corollary 4.5. Let $E$ be as in 4.4. If $T \in \operatorname{Orth}^{\sigma}(E)$ is invertible in $\operatorname{Orth}^{\infty}(E)$, then its inverse is a o-orthomorphism. 
COROllary 4.6. Let $E$ be as in 4.4 and assume moreover that $\operatorname{Orth}^{\sigma}(E)$ is rich. Then the following statements are equivalent:

(1) $\operatorname{Orth}^{\circ}(E)$ has the principal projection property;

(2) For every $T \in \operatorname{Orth}^{\sigma}(E)$ there exists $S \in \operatorname{Orth}^{\sigma}(E)$ such that $S T=\pi_{B_{T}}$;

(3) $\operatorname{Orth}^{\sigma}(E)$ is von Neumann regular.

Proof. It is easily seen that two extended orthomorphisms are disjoint if and only if they have disjoint supports; hence if $\mathbb{Q}=b(T)$ in $\operatorname{Orth}^{\sigma}(E)$, we have $\operatorname{Orth}^{\sigma}\left(E, B_{T}\right) \subseteq \mathbb{Q}$ and $\mathbb{Q}^{d}=\operatorname{Orth}^{\sigma}\left(E, B_{T}^{d}\right)$. But, if $\operatorname{Orth}^{\sigma}(E)$ is rich, we have also $Q=\operatorname{Orth}^{\sigma}\left(E, B_{T}\right)$ by virtue of 1.5 .

$(1) \Rightarrow(2)$. Let $T \in \operatorname{Orth}^{\sigma}(E)$; by (1), $Q=b(T)$ is a projection band and it follows from the above that $\pi_{B_{T}}$ must be the component of $I$ in $Q$. So $\pi_{B_{T}}$ is a $\sigma$-orthomorphism, that is, $B_{T} \oplus B_{T}^{d}$ is super order dense in $E$.

(2) $\Rightarrow$ (3), because $S T=\pi_{B_{T}}$ implies $S T^{2}=\pi_{B_{T}} T=T$.

(3) $\Rightarrow(1)$. Let $T \in \operatorname{Orth}^{\circ}(E)$ and $Q=b(T)$; by (3) there exists $S \in \operatorname{Orth}^{\circ}(E)$ such that $S T^{2}=T$, hence such that $T\left(\pi_{B_{T}}-S T\right)=0$. So, by 1.3, $\pi_{B_{T}}=S T \in$ $\operatorname{Orth}^{\sigma}(E)$ and, consequently, every $R \in \operatorname{Orth}^{\circ}(E)$ can be decomposed as $R=$ $\pi_{B_{T}} R+\pi_{B_{T}}^{d} R$ with $\pi_{B_{T}} R \in \mathcal{Q}$ and $\pi_{B_{T}}^{d} R \in \mathbb{Q}^{d}$. Hence $\mathcal{Q}$ is a projection band.

Remark. If $E$ is an almost Dedekind $\sigma$-complete Riesz space, then $\operatorname{Orth}^{\sigma}(E)$ is rich and has the principal projection property $[3 ; 3.4,3.6]$.

To conclude, we point out that a simple inspection of the proofs of 2.4 and 2.5 shows that it is possible to give an "extension theorem" for $\sigma$-orthomorphisms which is similar to 2.5 , but with more complicated hypothesis.

Theorem 4.7. Let $E$ be an Archimedean Riesz space, $F$ an order dense Riesz subspace of $E$ generating a super order dense ideal, and assume there exists a super order dense ideal $A$ of $E$ such that:

(1) $F \cap A$ is super order dense in $F$;

(2) $A$ is an $f$-algebra and $F \cap A$ is super quasi-unital.

Then the f-isomorphism $\psi: \operatorname{Orth}^{\infty}(F) \rightarrow \operatorname{Orth}^{\infty}(E)$ defined in 2.5 satisfies $\psi\left(\operatorname{Orth}^{\circ}(F)\right) \subseteq \operatorname{Orth}^{o}(E)$. Moreover, for any $T \in \operatorname{Orth}^{\circ}(F), T=\psi(T)$ holds on some super order dense ideal of $F$.

REMARK. If $E$ is uniformly complete and $F_{+}$has a maximal disjoint system $\left(e_{\alpha}\right)$ such that, for every $x \in F_{+},\left\{\alpha ; e_{\alpha} \wedge x \neq 0\right\}$ is at most countable, then $E$ has a super order dense ideal $A$ satisfying (1) and (2) of 4.7. 
EXAmPle. Given an Archimedean Riesz space $E$ and an order dense Riesz subspace $F$, it can happen that every $T \in \operatorname{Orth}^{\infty}(F)$ has a (unique) extension $\tilde{T} \in \operatorname{Orth}^{\infty}(E)$, but that $\tilde{T} \notin \operatorname{Orth}^{\sigma}(E)$ for some $T \in \operatorname{Orth}^{\sigma}(F)$ (even if $F$ is an ideal, in which case $\operatorname{Orth}^{\infty}(F)=\operatorname{Orth}^{\infty}(E)$ by 1.1). Let $X$ be a non countable set and $\mathscr{F}(X)$ the Riesz space of all real functions on $X$. If $E=\{f \in \mathscr{F}(X) ;\{f \neq a\}$ is at most countable for some $a \in \mathbf{R}\}$ and $F=\bigodot_{0}(X)$ (functions vanishing at $\infty$ ), then we have $\operatorname{Orth}^{\circ}(F)=\operatorname{Orth}^{\infty}(F)=\mathscr{F}(X)$, but $\operatorname{Orth}^{\circ}(E)=E$ and $\operatorname{Orth}^{\infty}(E)$ $=\mathscr{F}(X)$ (all these functions operating by product).

\section{References}

[1] C. D. Aliprantis and O. Burkinshaw, Locally solid Riesz spaces (Academic Press, New York-San Francisco-London, 1978)

[2] M. Duhoux and M. Meyer, 'A new proof of the lattice structure of orthomorphisms', J. London Math. Soc. (2) 25 (1982), 375-378.

[3] M. Duhoux and M. Meyer, 'Extended orthomorphisms on Archimedean Riesz spaces', Annali di Matematica (4) 133 (1983), 193-226.

[4] M. Duhoux and M. Meyer, 'Extended orthomorphisms and lateral completions of Archimedean Riesz spaces', to appear.

[5] C. B. Huijmans and B. de Pagter, 'Ideal theory in f-algebras', Trans. Amer. Math. Soc. 269 (1982), 225-245.

[6] W. A. J. Luxemburg, Some aspects of the theory of Riesz spaces (Lecture Notes in Mathematics 4 University of Arkansas, Fayetteville, 1979).

[7] W. A. J. Luxemburg and A. R. Schep, 'A Radon-Nikodym type theorem for positive operators and a dual', Nederl. Akad. Wetensch. Proc. Ser. A 81 (1978), 357-375.

[8] W. A. J. Luxemburg and A. C. Zaanen, Riesz spaces I (North-Holland, Amsterdam-London, 1971).

[9] M. Meyer, 'Richesses du centre d'un espace vectoriel réticulé', Math. Ann. 236 (1978) 147-169.

[10] M. Meyer, 'Les algèbres réticulées archimédiennes', Rev. Roumaine Math. Pures et Appl., to appear.

[11] H. Nakano, 'Teilweise geordnete Algebra', Japan J. Math. 17 (1941), 425-511.

[12] B. de Pagter, f-algebras and orthomorphisms (Thesis, University of Leiden, 1981).

[13] B. de Pagter, 'The space of extended orthomorphisms in a Riesz space', to appear.

[14] H. H. Schaefer, Banach lattices and positive operators (Springer-Verlag, Berlin-Heidelberg-New York, 1974).

[15] B. Z. Vulikh, Intraduction to the theory of partially ordered spaces (Wolters-Noordhoff Scientific Publ. Ltd, Groningen, 1967).

[16] A. W. Wickstead, 'Extensions of orthomorphisms', J. Austral. Math. Soc. Ser. A 29 (1980), 87-98.

Université Catholique de Louvain

Institut de Mathématique Pure et

Appliquée

Chemin du Chclotron

1348 Louvain-la-Neuve

Equipe d'Analyse Université Paris VI

Tour $464^{\mathrm{eme} e}$ etage

4, Place Jussieu

Belgique 75230 Paris-Cedex 05

France 